\title{
La longue quête des « Amis de l'Atlantide »
}

\section{Laurence Perry}

\section{(2) OpenEdition}

\section{Journals}

Édition électronique

URL : https://journals.openedition.org/rbnu/2855

DOI : $10.4000 /$ rbnu. 2855

ISSN : 2679-6104

\section{Éditeur}

Bibliothèque nationale et universitaire de Strasbourg

\section{Édition imprimée}

Date de publication : 1 mai 2012

Pagination : 72-77

ISSN : 2109-2761

\section{Référence électronique}

Laurence Perry, "La longue quête des « Amis de l'Atlantide » », La Revue de la BNU [En ligne], 5 | 2012 , mis en ligne le 01 mai 2012, consulté le 25 août 2021. URL : http://journals.openedition.org/rbnu/2855 ; DOI : https://doi.org/10.4000/rbnu.2855

Ce document a été généré automatiquement le 25 août 2021.

\section{(c) (i) (2) (2)}

La Revue de la BNU est mise à disposition selon les termes de la Licence Creative Commons Attribution - Pas d'Utilisation Commerciale - Partage dans les Mêmes Conditions 4.0 International. 


\title{
La longue quête des « Amis de l'Atlantide »
}

\author{
Laurence Perry
}

Dans le fonds d'archives «Edouard Schuré » conservé aux Archives de la Ville et de la Communauté urbaine de Strasbourg, parmi des dossiers consacrés au symbolisme et à la théosophie, figurent des tracts et un bulletin, datés de 1927, concernant une association qui venait d'être fondée: les «Amis de l'Atlantide "1. Ses initiateurs affirment rechercher le continent disparu mais également un idéal spirituel perdu, le retour à une tradition préchrétienne de sagesse et de beauté. Outre des causeries, dîners et excursions, les Amis de l'Atlantide annoncent deux revues : La nouvelle revue et Atlantis. Des comptes rendus de tous les ouvrages se rapportant aux questions atlantidéennes (ou atlantéennes) doivent paraître dans La nouvelle revue.

L'Atlantide, civilisation insulaire, est évoquée par Platon, au $4^{\mathrm{e}}$ siècle avant Jésus-Christ, dans deux de ses dialogues, le Timée et le Critias. Selon une tradition transmise par Solon, une île aurait été engloutie à l'ère préchrétienne vers 9000 avant Jésus-Christ, quelque part près du détroit de Gibraltar. Ses habitants auraient développé une civilisation brillante et un système politique remarquable. Simple métaphore, d'après Pierre Vidal-Naquet ${ }^{2}$, mais le mythe est né et fait l'objet, depuis plus de 2000 ans, de nombreuses spéculations quant à sa véracité et à l'emplacement de l'île engloutie, suscitant des dizaines de milliers de publications à visées scientifiques ${ }^{3}$.

À la Renaissance, l'intérêt pour l'Atlantide reprend vigueur avec l'engouement pour l'Antiquité et Platon, mais aussi grâce à la découverte d'un monde jusqu'alors inconnu, l'Amérique, dans laquelle certains voient justement l'Atlantide. À partir du $17^{\mathrm{e}}$ siècle, les théories pseudo-scientifiques sur l'emplacement de l'Atlantide foisonnent.

Aux $19^{\mathrm{e}}$ et $20^{\mathrm{e}}$ siècles, deux grands succès d'édition popularisent l'Atlantide : Vingt mille lieues sous les mers de Jules Vernes, paru en 1869 chez Hetzel, dans lequel les héros découvrent l'Atlantide, et surtout, en 1919, chez Albin Michel, le roman de Pierre Benoit, L'Atlantide. Pour l'auteur, qui situe l'Atlantide au Sahara, il s'agit d'une pure fiction, mais il relance l'intérêt d'un large public pour la cité engloutie. Dopée par la 
quête de l'âge d'or, d'un paradis perdu et par la vogue de l'occultisme et de l'ésotérisme, la littérature consacrée à l'Atlantide connaît une inflation inouïe.

Page de titre de l'édition originale du roman de Pierre Benoit

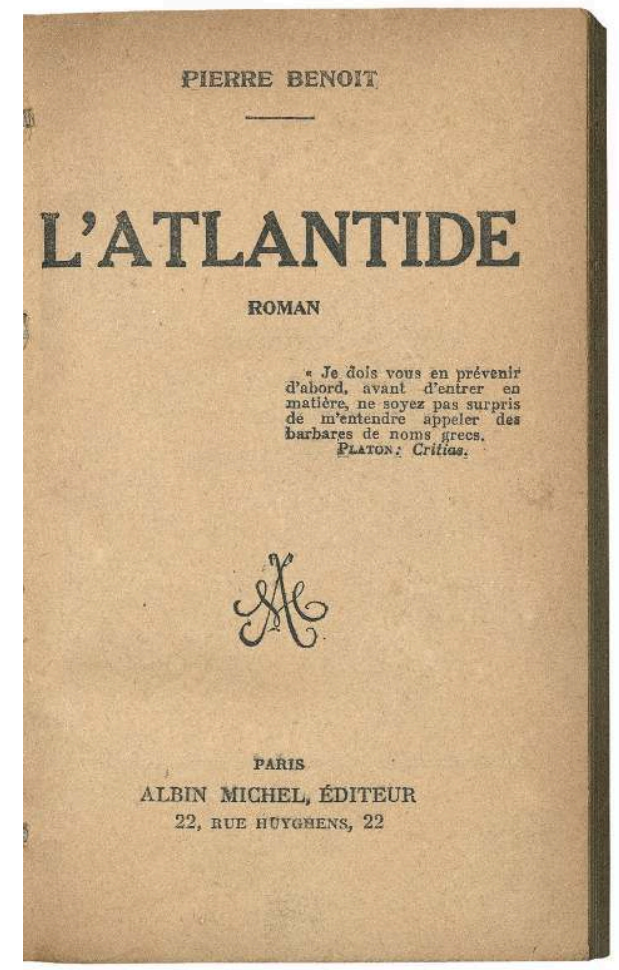

Cliché JPR-Bnu CC-BY-NC-SA

5 À l'orée du $20^{\mathrm{e}}$ siècle, les chercheurs de divers horizons, archéologues, géologues, géographes, historiens, cherchent à établir si les progrès de la recherche et de l'exploration sous-marine permettent de corroborer ou d'infirmer l'existence d'une civilisation perdue. Mais d'autres personnages, qui n'appartiennent pas au monde scientifique, sont fascinés par le récit de Platon. Le journaliste et romancier Roger Dévigne (1885-1965) publie en 1923 Voyages. Un continent disparu, l'Atlantide, sixième partie du monde. Il se fonde sur les travaux scientifiques en cours. De son côté, en 1925, l'écrivain ésotérique Paul Le Cour fait paraître dans le Mercure de France plusieurs articles sur l'Atlantide: il cherche, à travers ce mythe, à reconstruire une tradition européenne. Les deux hommes s'associent pour créer à la Sorbonne la "Société d'études atlantéennes ", le 24 juin 1926. Les divergences de vues entre Dévigne et Le Cour entraînent la démission de ce dernier en 1927. Les deux hommes s'affrontent par presse interposée puis Le Cour crée sa propre société, les Amis de l'Atlantide".

Quelques notes de la main de Schuré accompagnent les tracts des Amis de l'Atlantide. Il a recopié un passage d'un article de Georges Gourdon dans la Revue littéraire et artistique de 1885, évoquant successivement les théories de Bory de Saint-Vincent (qui, dans son ouvrage Essais sur les isles Fortunées et l'antique Atlantide, paru en 1803, situait la cité disparue vers les Canaries et les Açores) $)^{5}$, la campagne du Talisman dans la mer des Sargasses et des Açores en $1883^{6}$ ainsi que l'épopée de Jacinto Verdaguer L'Atlantide ${ }^{7}$. "Quelques mots sur l'Atlantide», notes griffonnées, expliquent la nature philosophique et religieuse du mouvement atlantéen, «qui s'appuie sur des données 
scientifiques ", énonce Schuré. L'humanité du début du $20^{\mathrm{e}}$ siècle, qui a sombré dans "le matérialisme intellectuel et l'anarchie individualiste », a perdu "le sens du divin, de ses origines et de son but ».

7 Edouard Schuré cultivait un intérêt personnel pour l'Atlantide, qui fait l'objet du livre II de L'évolution divine du Sphinx au Christ, publié en 1912. À son habitude, Schuré mêle connaissances scientifiques et poésie. Du coup, l'écrivain et journaliste littéraire Antoine Albalat ironise sur sa "somptueuse description des habitants de l'Atlantide: on finit par connaître les Atlantes comme si on les avait fréquentés ${ }^{8}$. Schuré, comme Steiner, à qui l'ouvrage est d'ailleurs dédié, croit que l'Atlantide est le berceau de la race blanche ${ }^{9}$. Il décède en 1929 , deux ans après la création de l'association, qui laissa donc peu de traces dans ses archives. Je la croyais sans doute disparue, mais en écrivant son nom sur un moteur de recherche, je retrouvai la trace de ses débuts et la confirmation de l'existence de la revue. Plus encore, je m'aperçus, à ma grande surprise, que l'association, toujours active, disposait d'un site internet à jour ${ }^{10}$, n'avait jamais cessé de publier, ni de faire des conférences à Paris et à Lyon.

Notes de la main d'Edouard Schuré sur l'Atlantide 1927

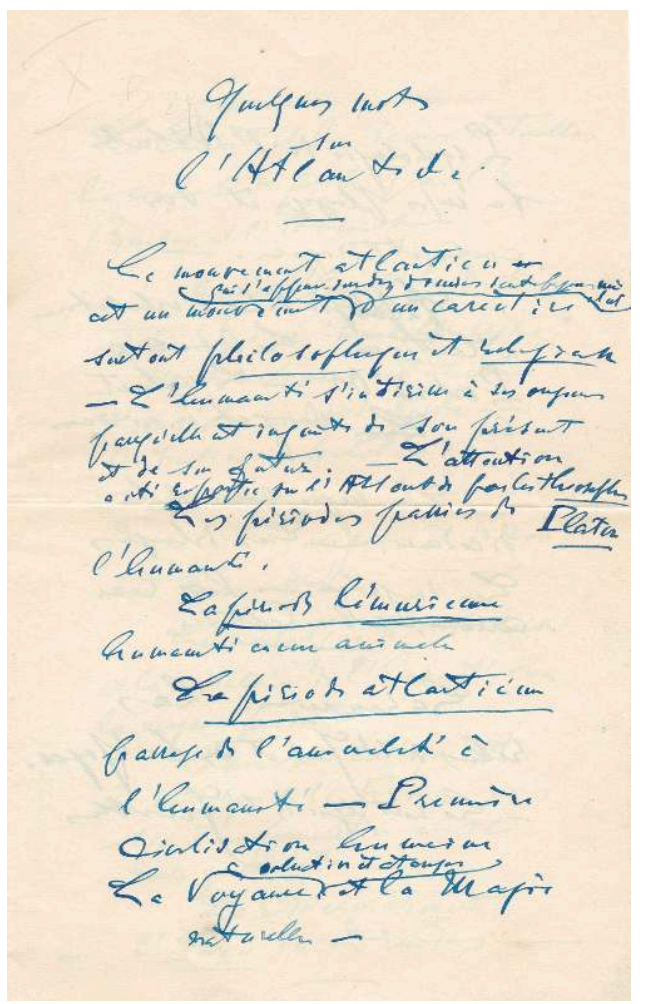

Clichés Stéphane Arena, Archives de Strasbourg 11 Z 108

La revue Atlantis se trouve être partiellement conservée à la Bibliothèque nationale et universitaire de Strasbourg où j'entrepris de la dépouiller. Cela me renvoya de nouveau à Schuré : l'écrivain a figuré un temps dans le comité d'honneur de l'association! Je pensai alors qu'en raison de la convergence de leurs centres d'intérêt, il était fort possible que des membres et sympathisants aient été en relations épistolaires avec l'écrivain. Il s'avéra que les écrivains Pierre Lecomte du Nouy ${ }^{11}$, Victor-Émile Michelet ${ }^{12}$, J.-H. Rosny aîné ${ }^{13}$, Aimé Rutot ${ }^{14}$ (de l'Académie royale de Belgique), Fortunat Strowski ${ }^{15}$ 
et Jacques-Treve (en fait une femme, amie des fondateurs de la revue) figuraient à la fois parmi les membres du comité d'honneur et parmi ses correspondants.

Le but des Amis de l'Atlantide, tel qu'il est annoncé dans leur revue, est de tendre « à rechercher, à travers les légendes, les symboles, les religions et les philosophies, la Tradition primitive, sur laquelle il est nécessaire de s'appuyer pour obtenir une rénovation spirituelle permettant de préparer les Temps nouveaux vers lesquels l'humanité marche à grands pas $»^{16}$.

Où se place l'Atlantide dans ce vaste dessein? Le principal fondateur de l'association, Paul Le Cour, y situait le berceau de toutes les civilisations. Il voulait revenir à un idéal perdu, rêve d'une nouvelle chevalerie. Pour lui, « rechercher l'Atlantide, c'est faire les premiers pas vers la constitution des Etats-Unis d'Europe $»^{17}$ avec une charte spirituelle commune. Il signale fièrement que deux collaborateurs de la revue Notre Europe fondée à 1951 à Strasbourg, Louis Rougier et la duchesse de La Rochefoucauld, sont abonnés à Atlantis. Les personnalités peu banales à l'origine de l'association expliquent la dimension idéaliste prise par les Amis de l'Atlantide et sans doute la longévité de leur mouvement. Paul Le Cour (1871-1954), après des études classiques, renonce à une vocation de marin, contrariée par la volonté maternelle, et devient percepteur pendant seize ans. Parallèlement à ces fonctions bien terre à terre, il passe dix ans à étudier les phénomènes spirites et métapsychiques. Artiste, il s'adonne aussi à la musique, l'aquarelle, la photographie. Professionnellement, il aboutit en 1905 au ministère des Travaux publics à Paris. Fonctionnaire passablement original, il transforme son service en « colonie platonicienne », selon un de ses amis ${ }^{18}$.

Tract annonçant la fondation de l'association « Les Amis de l'Atlantide », 1927

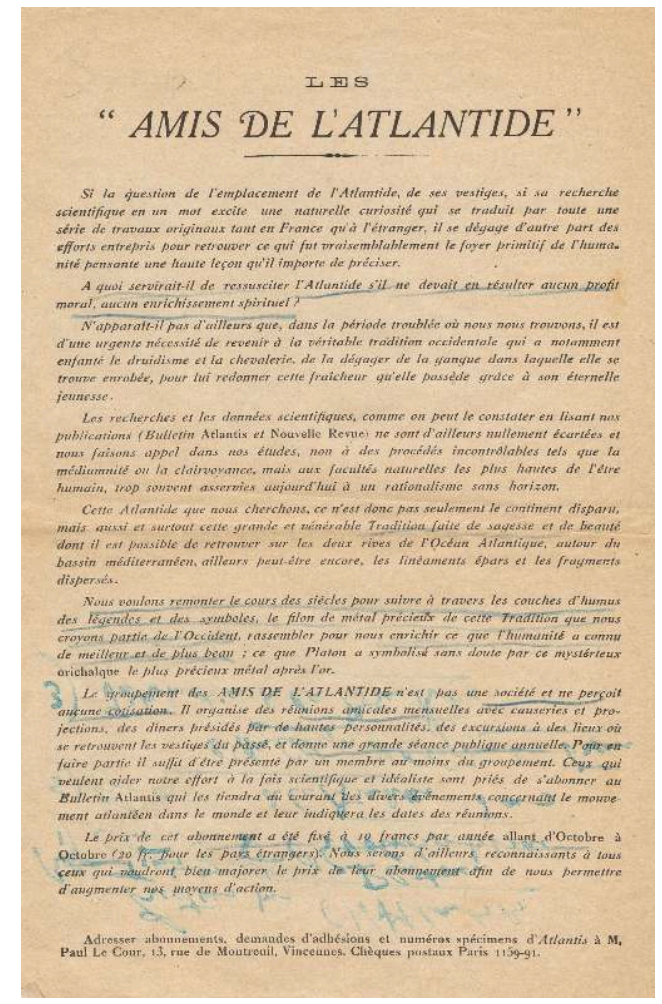

Clichés Stéphane Arena, Archives de Strasbourg 11 Z 108 
11 Le deuxième cofondateur, Philéas Lebesgue (1869-1958), agriculteur, écrivain, rédacteur au Mercure de France, polyglotte, grand druide de la Gorsedd de Bretagne, est encore plus singulier. Son œuvre sera couronnée trois fois par l'Académie française. Il collabore de 1928 à 1942 à Atlantis. Pour Lebesgue, l'Atlantide s'avère plus un idéal qu'une réalité tangible alors que Le Cour croit fermement à son existence. Enfin, François Roussel Despierres (1864-après 1936), conseiller d'État, secrétaire d'État de la principauté de Monaco, auteur de nombreux ouvrages et d'études parues dans La nouvelle revue, complète le trio.

Leur revue, Atlantis, se fait bien entendu l'écho des découvertes et thèses à propos de l'Atlantide qui sont commentées et souvent démontées. En 1952, une contribution de René Malaise, membre du Museum suédois d'histoire naturelle, situant l'Atlantide dans les Açores, est signalée. Dans le numéro 159, Paul Le Cour critique une théorie de l'autrichien Hoerbiger, qui situe l'Atlantide au Pérou. Elle aurait disparu après la collision entre la Terre et une petite planète. Toutefois, Le Cour admet que les Atlantéens aient pu émigrer au Pérou. Le numéro 162 se fait l'écho de la découverte de l'Atlantide en mer du Nord par le pasteur Spanuth, dans les parages de l'île d'Héligoland. L'écrivain Claude Farrère ${ }^{19}$ (1876-1957) s'enflamme dans le n 163 : «Je crois ne jamais me tromper en donnant toujours raison aux poètes contre les savants. Les poètes se trompent infiniment moins souvent que les manieurs d'intégrales. Je donne raison à Platon et je donne raison à Jules Verne. Je crois à l'Atlantide, je crois que les îles du Cap-Vert, que Madère, que les Açores et les Canaries en sont les derniers vestiges $»^{20}$. Les progrès de l'exploration sous-marine font naître bien des espoirs : le numéro 170 mentionne les expéditions du commandant Cousteau et de la Calypso ! Le numéro 213, en 1962, titre Symbolisme et Atlantide; le 336, en 1985, porte sur «l'Atlantide face à la science ». La même année, l'association organise un séminaire intitulé «L'Atlantide, une quête toujours recommencée », rassemblant océanographes, historiens, journalistes pour des expositions, colloques, diaporamas. Des divergences apparaissent alors entre les tenants du nord du Sahara et ceux des Canaries. 


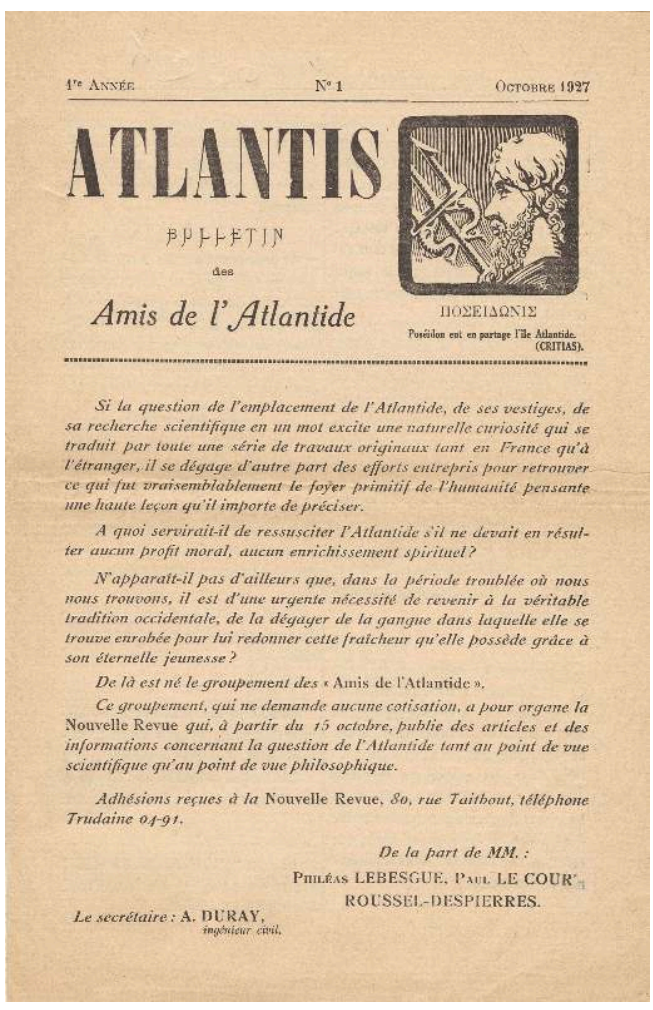

Clichés Stéphane Arena, Archives de Strasbourg 11 Z 108

13 Mais les Amis de l'Atlantide ne consacrent pas leurs travaux, depuis quatre-vingt-dix ans, uniquement à l'Atlantide du point de vue archéologique. Leurs préoccupations rejoignent tout à fait celles d'Edouard Schuré : symbolisme, celtisme, ésotérisme. Plusieurs cathédrales, dont celle de Strasbourg, sont étudiées sous l'angle symbolique. De nombreux numéros traitent du celtisme, des druides et les Amis de l'Atlantide s'associent en 1955 au bimillénaire de la mort de Vercingétorix ${ }^{21}$ en consacrant un numéro à Gergovie. En musique, la Suite atlante de Claude Guiraud et l'Atlantida de Manuel de Falla sont analysées ${ }^{22}$ en même temps que l'œuvre de Wagner ${ }^{23}$. Un numéro est dédié au symbolisme des nombres, un autre au symbolisme architectural. Le peintre Gustave Moreau fait l'objet d'une revue en 1965 mais également les Etrusques, peuple disparu ${ }^{24}$, la Rose-Croix et les rosicruciens ${ }^{25}$. L'occultisme, par contre, n'a pas vraiment les faveurs de la revue : "La Tradition, salut du monde, l'occultisme, fléau du monde », écrivait Paul Le Cour ${ }^{26}$.

Le dernier numéro consacré à l'Atlantide que possède la BNU remontant à $1994^{27}$, Gérald Scozzari, rédacteur en chef de la revue, accepta aimablement de satisfaire ma curiosité quant à l'actualité de la recherche atlantidéenne, dont il est le référent au sein de l'association. Les Amis de l'Atlantide suivent avec intérêt le progrès des découvertes dans le Sud de l'Espagne, la région du Guadalquivir. Tartessos, civilisation antérieure aux Phéniciens, pourrait avoir suscité le mythe de l'Atlantide. De fait, un documentaire du National Geographic, intitulé Finding Atlantis et daté de mars 2011 évoque un lien entre la civilisation enfouie sous les sables et l'Atlantide. Mentionnée par de nombreux auteurs anciens grecs et latins, cette civilisation florissante disparue il y a environ 3000 ans aurait été détruite par une catastrophe naturelle, tremblement de terre et tsunami. Plusieurs chercheurs ont alors fait le lien avec le mythe de l'Atlantide. Les fouilles 
conduites par les Espagnols permettront-elles de mettre fin à la quête de l'île disparue ? Nous pouvons en douter car l'imagination, le rêve et la poésie tiennent aussi toute leur place dans la recherche de la cité mythique.

\section{NOTES}

1. Archives de Strasbourg, $11 \mathrm{Z} 108$

2. L'Atlantide, petite histoire d'un mythe platonicien, Paris, Belles lettres, 2005

3. Lauric Guillaud, maître de conférences à l'université de Nantes, estime leur nombre à 40000 dans son étude Le cercle de l'éternel déluge (in Atlantides : les îles englouties, Paris, Omnibus, 1995).

4. Pierre Lagrange, Les controverses sur l'Atlantide (1925-1940). L'archéologie entre vraie et fausse science, in Ethnologie de la France, cahier $n^{\circ} 22,2008$

5. Jean-Baptiste Bory de Saint-Vincent (1778-1846), officier de marine, naturaliste et géographe

6. Il s'agit des premières campagnes scientifiques sous-marines françaises.

7. Jacinto Verdaguer (1845-1902), poète catalan, a publié en 1876 un poème, L'Atlantide.

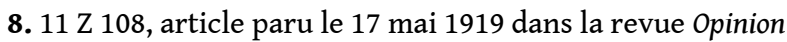

9. Cette croyance sera partagée par certains nazis.

10. https://www.association-atlantis.org/

11. Mathématicien, écrivain et philosophe français (1883-1947)

12. Poète ésotérique français (1861-1938), martiniste

13. Ecrivain d'origine belge (1856-1940), un des fondateurs de la science-fiction, auteur entre autres de La Guerre du feu

14. Aimé-Louis Rutot (1847-1933), géologue et préhistorien belge

15. Historien de la littérature et critique littéraire français (1866-1952), professeur à la Faculté des lettres de Paris

16. Atlantis, 1958

17. Atlantis, $\mathrm{n}^{\circ} 58$, mars 1935

18. Louis Rougier, in Atlantis, $\mathrm{n}^{\circ} 171$, p. 135

19. Pseudonyme de Frédéric-Charles Bargone (1876-1957), ancien officier de marine, prix Goncourt 1905 avec Les Civilisés

20. Page 178

21. Schuré avait publié en 1887 un drame en cinq actes, Vercingétorix.

22. Atlantis, $\mathrm{n}^{\circ} 182$

23. Atlantis, $\mathrm{n}^{\circ} 2031$

24. Atlantis, $\mathrm{n}^{\circ} \mathrm{s} 371,1992$ et 373,1993

25. Atlantis, $\mathrm{n}^{\circ \mathrm{s}} 233-235$

26. Atlantis, $\mathrm{n}^{\circ} 364,1991$

27. Atlantis, $\mathrm{n}^{\circ} 377$ 


\section{AUTEUR}

\section{LAURENCE PERRY}

Directrice des Archives de la Ville et de la Communauté urbaine de Strasbourg 